\title{
Second moment analysis for Robin boundary value problems on random domains
}

\author{
H. Harbrecht
}

Institute of Mathematics

Preprint No. 2012-13

University of Basel

August, 2012

Rheinsprung 21

CH - 4051 Basel

Switzerland 


\title{
Second moment analysis for Robin boundary value problems on random domains
}

\author{
H. Harbrecht
}

\begin{abstract}
We consider the numerical solution of Robin boundary value problems on random domains. The proposed method computes the mean and the variance of the random solution with leading order in the amplitude of the random boundary perturbation relative to an unperturbed, nominal domain. The variance is computed as the trace of the solution's two-point correlation which satisfies a deterministic boundary value problem on the tensor product of the nominal domain. We solve this moderate high-dimensional problem by either a low-rank approximation by means of the pivoted Cholesky decomposition or the combination technique. Both approaches are presented and compared by numerical experiments with respect to their efficiency.
\end{abstract}

\section{Introduction}

Many problems in physics and engineering sciences lead to boundary value problems for an unknown function. In general, the numerical simulation is well understood provided that the input parameters are given exactly. Since, however, exact input parameters are often not known in engineering, it is of growing interest to model such parameters as random variables.

A principal approach to solve boundary value problems with random input parameters is the Monte Carlo approach, see e.g. [37] and the references therein. However, it is hard and extremely expensive to generate a large number of suitable samples and to solve a deterministic boundary value problem on each sample. Particularly in the present case of random domains, each new sample corresponds to a new domain which needs to be discretized. Thus, we aim here at a direct, deterministic method to compute the random solution.

Helmut Harbrecht

Mathematisches Institut, Universität Basel, Rheinsprung 21, 4051 Basel, Switzerland

e-mail: helmut.harbrecht@unibas.ch 
Deterministic approaches to solve stochastic partial differential equations have been proposed in e.g. [1, 11, 13, 14, 25, 32, 38]. Therein, loadings and coefficients have been considered as random input parameters. Recently, in [6, 23, 27, 33, 34, 43], also the underlying domain has been modeled as a random input parameter $D(\omega)$. For example, this enables the consideration of tolerances in the shape of products fabricated by line production. Other applications arise from blurred interfaces like cell membranes or molecular surfaces.

The present paper is dedicated to the numerical treatment of Robin boundary value problems on random domains which, to the best of our knowledge, is the first time in the scientific literature. We assume small random perturbations around a nominal domain $\bar{D}$ with known second order statistics. Then, following [27], we can linearize to derive, with leading order in the amplitude of the perturbation parameter, deterministic equations for the random solution's expectation and two-point correlation

$$
\left.\begin{array}{rl}
\mathbb{E}_{u}(\mathbf{x}) & =\int_{\Omega} u(\mathbf{x}, \omega) \mathrm{d} P(\omega) \\
\operatorname{Cor}_{u}(\mathbf{x}, \mathbf{y}) & =\int_{\Omega} u(\mathbf{x}, \omega) u(\mathbf{y}, \omega) \mathrm{d} P(\omega)
\end{array}\right\} \quad \mathbf{x}, \mathbf{y} \in \bar{D} .
$$

From these quantities the variance is derived by

$$
\mathbb{V}_{u}(\mathbf{x})=\left.\operatorname{Cor}_{u}(\mathbf{x}, \mathbf{y})\right|_{\mathbf{x}=\mathbf{y}}-\mathbb{E}_{u}^{2}(\mathbf{x}), \quad \mathbf{x} \in \bar{D} .
$$

The solution's two-point correlation is given by a partial differential equation which lives on the tensor product domain $\bar{D} \times \bar{D}$. We solve this moderate highdimensional problem by either a low-rank approximation via the pivoted Cholesky decomposition or the combination technique which is a special variant of a sparse tensor product approximation. This way, we are able to compute both, the expectation and the variance by standard finite element techniques.

Besides the modeling and the derivation of the underlying equations, we discuss in this paper the implementation of the proposed algorithms. In particular, we compare the low-rank approximation and the tensor product approximation with respect to their cost-complexities by numerical results.

The rest of the paper is organized as follows. In Section 2, we model the random domain under consideration. Moreover, for the associated Robin boundary value problem, we derive deterministic boundary value problems for the expectation and two-point correlation of the random solution. In Section 3, we introduce the variational formulations of these deterministic boundary value problems. Section 4 is dedicated to an abstract overview on the efficient solution of tensor product-type boundary value problems which arise in the present context. The particular finite element discretization of the problems under consideration are performed in Section 5. In Section 6, numerical experiments are carried out to validate the theoretical findings and to compare the low-rank approximation with the sparse grid approach. Finally, in Section 7, we state concluding remarks. 


\section{Robin boundary value problems on random domains}

Let $(\Omega, \Sigma, P)$ be a suitable probability space. We consider the domain $D(\omega)$ as the uncertain input parameter of an elliptic boundary value problem with Robin boundary conditions, i.e.,

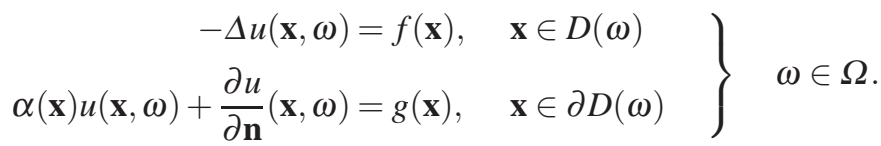

Here, $\alpha(\mathbf{x}) \geq 0$ is a nonnegative function, where the particular choice $\alpha(\mathbf{x}) \equiv 0$ yields the Neumann boundary condition.

To model the random domain $D(\omega)$, let $\bar{D}$ denote a smooth reference domain and consider random boundary variations in the direction of the outer normal

$$
\mathbf{U}(\mathbf{x}, \omega)=\varepsilon \kappa(\mathbf{x}, \omega) \mathbf{n}(\mathbf{x}): \partial \bar{D} \rightarrow \mathbb{R}^{n}
$$

with

$$
\kappa(\omega) \in L_{P}^{2}\left(\Omega, C^{2,1}(\partial \bar{D})\right) \quad \text { such that }\|\kappa(\omega)\|_{C^{2,1}(\partial \bar{D})} \leq 1
$$

almost surely. Then, the random domain $D(\omega)$ will be described via perturbation of identity

$$
\partial D(\omega)=\{(\mathbf{I}+\varepsilon \mathbf{U}(\omega))(\mathbf{x})=\mathbf{x}+\varepsilon \kappa(\mathbf{x}, \omega) \mathbf{n}(\mathbf{x}): \mathbf{x} \in \partial \bar{D}\} .
$$

For what follows we assume that the expectation $\mathbb{E}_{\kappa}$ and the two-point correlation Cor $_{\kappa}$ of the boundary perturbation $\kappa$ are given. Without loss of generality (otherwise we redefine $\bar{D}$ correspondingly) we assume that the perturbation field $\kappa$ is centered, i.e., that $\mathbb{E}_{\kappa} \equiv 0$.

For a small perturbation amplitude $\varepsilon>0$, one can linearize (1) by means of shape calculus $[12,40]$. This leads to the following stochastic shape-Taylor expansion

$$
u(\mathbf{x}, \omega)=\bar{u}(\mathbf{x})+\varepsilon \delta u[\kappa(\omega)](\mathbf{x})+\mathscr{O}\left(\varepsilon^{2}\right), \quad \mathbf{x} \in K \Subset \bar{D} .
$$

Therein, the compact set $K \Subset \bar{D}$ is assumed to satisfy $K \Subset D(\omega)$ almost surely. Moreover, $\bar{u} \in H^{1}(\bar{D})$ denotes the solution to the deterministic Robin boundary value problem

$$
\begin{aligned}
& -\Delta \bar{u}(\mathbf{x})=f(\mathbf{x}), \quad \mathbf{x} \in \bar{D} \\
& \alpha(\mathbf{x}) \bar{u}(\mathbf{x})+\frac{\partial \bar{u}}{\partial \mathbf{n}}(\mathbf{x})=g(\mathbf{x}), \quad \mathbf{x} \in \partial \bar{D}
\end{aligned}
$$

and the shape derivative $\delta u=\delta u[\kappa] \in H^{1}(\bar{D})$ satisfies the following Robin boundary value problem with random loading (cf. [31])

$$
\begin{aligned}
\Delta \delta u(\mathbf{x}) & =0, & & \mathbf{x} \in \bar{D} \\
\alpha(\mathbf{x}) \delta u(\mathbf{x})+\frac{\partial \delta u}{\partial \mathbf{n}}(\mathbf{x}) & =\operatorname{div}_{\Gamma}\left(\kappa(\mathbf{x}) \nabla_{\Gamma} \bar{u}(\mathbf{x})\right)+\kappa(\mathbf{x}) h(\mathbf{x}), & & \mathbf{x} \in \partial \bar{D} .
\end{aligned}
$$


Here, we used the abbreviation

$$
h(\mathbf{x}):=f(\mathbf{x})+\mathscr{H}(\mathbf{x})(g(\mathbf{x})-\alpha(\mathbf{x}) \bar{u}(\mathbf{x}))+\frac{\partial(g-\alpha \bar{u})}{\partial \mathbf{n}}(\mathbf{x}),
$$

where $\mathscr{H}=(n-1) \overline{\mathscr{H}}$ is the additive curvature and $\overline{\mathscr{H}}$ is the mean curvature of the surface $\Gamma$.

Theorem 1. Assume that the compact set $K \Subset \bar{D}$ satisfies $K \Subset D(\omega)$ almost surely. Then, it holds that

$$
\left.\begin{array}{l}
\mathbb{E}_{u}(\mathbf{x})=\bar{u}(\mathbf{x})+\mathscr{O}\left(\varepsilon^{2}\right) \\
\mathbb{V}_{u}(\mathbf{x})=\left.\varepsilon^{2} \operatorname{Cor}_{\delta u}(\mathbf{x}, \mathbf{y})\right|_{\mathbf{x}=\mathbf{y}}+\mathscr{O}\left(\varepsilon^{3}\right)
\end{array}\right\} \quad \mathbf{x} \in K
$$

Herein, $\bar{u} \in H^{1}(\bar{D})$ and $\operatorname{Cor}_{\delta u}(\mathbf{x}, \mathbf{y}) \in H_{m i x}^{1}(\bar{D} \times \bar{D}):=H^{1}(\bar{D}) \times H^{1}(\bar{D})$ satisfy the deterministic boundary value problems (3) and

$$
\begin{gathered}
\left(\Delta_{\mathbf{x}} \otimes \Delta_{\mathbf{y}}\right) \operatorname{Cor}_{\delta u}(\mathbf{x}, \mathbf{y})=0, \quad \mathbf{x}, \mathbf{y} \in \bar{D} \\
\Delta_{\mathbf{x}} \operatorname{Cor}_{\delta u}(\mathbf{x}, \mathbf{y})=0, \quad \mathbf{x} \in \bar{D}, \mathbf{y} \in \partial \bar{D}, \\
\Delta_{\mathbf{y}} \operatorname{Cor}_{\delta u}(\mathbf{x}, \mathbf{y})=0, \quad \mathbf{x} \in \partial \bar{D}, \mathbf{y} \in \bar{D} \\
{\left[\left(\alpha(\mathbf{x})+\frac{\partial}{\partial \mathbf{n}_{\mathbf{x}}}\right) \otimes\left(\alpha(\mathbf{y})+\frac{\partial}{\partial \mathbf{n}_{\mathbf{y}}}\right)\right] \operatorname{Cor}_{\delta u}(\mathbf{x}, \mathbf{y})=\operatorname{Cor}_{\kappa}(\mathbf{x}, \mathbf{y})[h(\mathbf{x}) \otimes h(\mathbf{y})]} \\
+\operatorname{div}_{\Gamma, \mathbf{x}}\left[\operatorname{Cor}_{\kappa}(\mathbf{x}, \mathbf{y})\left(\nabla_{\Gamma} \bar{u}(\mathbf{x}) \otimes f(\mathbf{y})\right)\right]+\operatorname{div}_{\Gamma, \mathbf{y}}\left[\operatorname{Cor}_{\kappa}(\mathbf{x}, \mathbf{y})\left(h(\mathbf{x}) \otimes \nabla_{\Gamma} \bar{u}(\mathbf{y})\right)\right] \\
+\left(\operatorname{div}_{\Gamma, \mathbf{x}} \otimes \operatorname{div}_{\Gamma, \mathbf{y}}\right)\left[\operatorname{Cor}_{\kappa}(\mathbf{x}, \mathbf{y})\left(\nabla_{\Gamma} \bar{u}(\mathbf{x}) \otimes \nabla_{\Gamma} \bar{u}(\mathbf{y})\right)\right], \quad \mathbf{x}, \mathbf{y} \in \partial \bar{D} .
\end{gathered}
$$

Proof. By using the shape-Taylor expansion (2), we obtain

$$
\mathbb{E}_{u}(\mathbf{x})=\bar{u}(\mathbf{x})+\varepsilon \mathbb{E}(\delta u[\kappa(\omega)](\mathbf{x}))+\mathscr{O}\left(\varepsilon^{2}\right) .
$$

By the linearity of the expectation operator $\mathbb{E}$, taking the expectation on both sides of (4), and observing that $\mathbb{E}_{\kappa}(\mathbf{x}) \equiv 0$, we have $\mathbb{E}_{\delta u}(\mathbf{x})=\mathbb{E}(\delta u[\kappa(\omega)](\mathbf{x})) \equiv 0$, which yields the first claim.

Observe the following estimate

$$
\begin{aligned}
\mathbb{V}(a+b X+c Y) & =b^{2} \mathbb{V}(X)+2 b c \operatorname{Cov}(X, Y)+c^{2} \mathbb{V}(Y) \\
& \leq b^{2} \mathbb{V}(X)+2 b c \sqrt{\mathbb{V}(X) \mathbb{V}(Y)}+c^{2} \mathbb{V}(Y),
\end{aligned}
$$

where $X$ and $Y$ are two random variables with finite second moments. By combining this estimate with the shape-Taylor expansion (2), we conclude

$$
\begin{aligned}
\mathbb{V}_{u}(\mathbf{x}) & =\varepsilon^{2} \mathbb{V}(\delta u[\kappa(\omega)](\mathbf{x}))+\sqrt{\mathbb{V}(\delta u[\kappa(\omega)](\mathbf{x}))} \mathscr{O}\left(\varepsilon^{3}\right)+\mathscr{O}\left(\varepsilon^{4}\right) \\
& =\varepsilon^{2} \mathbb{V}_{\delta u}(\mathbf{x})+\mathscr{O}\left(\varepsilon^{3}\right) .
\end{aligned}
$$


Due to $\mathbb{E}_{\delta u}(\mathbf{x}) \equiv 0$, we arrive at the identity $\mathbb{V}_{\delta u}(\mathbf{x})=\left.\operatorname{Cor}_{\delta u}(\mathbf{x}, \mathbf{y})\right|_{\mathbf{x}=\mathbf{y}}$ which proves the second claim. The boundary value problem (7) for $\mathrm{Cor}_{\delta u}$ is finally derived by tensorizing (4) and taking the expectation. This completes the proof.

Remark 1. The relative error of the expectation is $\mathscr{O}\left(\varepsilon^{2}\right)$ while the relative error of the variance is $\mathscr{O}(\varepsilon)$. According to [8], the first order shape-Taylor expansion (2) is nevertheless sufficient to compute also higher order moments of the random solution with relative accuracy $\mathscr{O}(\varepsilon)$.

\section{Variational formulation}

We shall introduce the variational formulations of the boundary value problems under consideration. The approximate expectation $\bar{u} \in H^{1}(\bar{D})$, satisfying (3), is determined by the variational formulation

$$
\text { seek } \bar{u} \in H^{1}(\bar{D}) \text { such that } a(\bar{u}, v)=\ell_{1}(v) \text { for all } v \in H^{1}(\bar{D}),
$$

where the bilinear form $a: H^{1}(\bar{D}) \times H^{1}(\bar{D}) \rightarrow \mathbb{R}$ is given by

$$
a(u, v):=\int_{\bar{D}} \nabla u(\mathbf{x}) \nabla v(\mathbf{x}) \mathrm{d} \mathbf{x}+\int_{\partial \bar{D}} \alpha(\mathbf{x}) u(\mathbf{x}) v(\mathbf{x}) \mathrm{d} \sigma
$$

and the linear form $\ell_{1}: H^{1}(\bar{D}) \rightarrow \mathbb{R}$ by

$$
\ell_{1}(v):=\int_{\bar{D}} f(\mathbf{x}) v(\mathbf{x}) \mathrm{d} \mathbf{x}+\int_{\partial \bar{D}} g(\mathbf{x}) v(\mathbf{x}) \mathrm{d} \sigma .
$$

The shape derivative $\delta u=\delta u[\kappa] \in H^{1}(\bar{D})$ in a given direction $\kappa \in C^{2,1}(\partial \bar{D})$ satisfies the boundary value problem (4). The associated variational formulation involves the same bilinear form as (8), but a different linear form on the right hand side. Namely, we find

$$
\text { seek } \delta u \in H^{1}(\bar{D}) \text { such that } a(\delta u, v)=\ell_{2}(v) \text { for all } v \in H^{1}(\bar{D}),
$$

with the linear form $\ell_{2}: H^{1}(\bar{D}) \rightarrow \mathbb{R}$ being defined by

$$
\ell_{2}(v):=\int_{\partial \bar{D}} \kappa(\mathbf{x})\left\{h(\mathbf{x})-\nabla_{\Gamma} \bar{u}(\mathbf{x}) \nabla_{\Gamma}\right\} v(\mathbf{x}) \mathrm{d} \sigma .
$$

Note that we applied integration by parts in the definition of the linear form. Moreover, the function $h$ is defined (5). Thus, the two-point correlation function $\operatorname{Cor}_{\delta u} \in H_{m i x}^{1}(\bar{D} \times \bar{D})$, which is given by the tensor Robin boundary value problem (7), satisfies the variational formulation

$$
\begin{aligned}
& \text { seek } \operatorname{Cor}_{\delta u} \in H_{m i x}^{1}(\bar{D} \times \bar{D}) \text { such that } \\
& \qquad A\left(\operatorname{Cor}_{\delta u}, v\right)=L(v) \text { for all } v \in H_{m i x}^{1}(\bar{D} \times \bar{D}) .
\end{aligned}
$$


Here, the bilinear form $A: H_{\text {mix }}^{1}(\bar{D} \times \bar{D}) \times H_{\text {mix }}^{1}(\bar{D} \times \bar{D}) \rightarrow \mathbb{R}$ reads as

$$
\begin{aligned}
A(u, v):= & \int_{\bar{D}} \int_{\bar{D}}\left(\nabla_{\mathbf{x}} \otimes \nabla_{\mathbf{y}}\right) u(\mathbf{x}, \mathbf{y})\left(\nabla_{\mathbf{x}} \otimes \nabla_{\mathbf{y}}\right) v(\mathbf{x}, \mathbf{y}) \mathrm{d} \mathbf{y} \mathrm{d} \mathbf{x} \\
& +\int_{\bar{D}} \int_{\partial \bar{D}} \alpha(\mathbf{y}) \nabla_{\mathbf{x}} u(\mathbf{x}, \mathbf{y}) \nabla_{\mathbf{x}} v(\mathbf{x}, \mathbf{y}) \mathrm{d} \sigma_{\mathbf{y}} \mathrm{d} \mathbf{x} \\
& +\int_{\partial \bar{D}} \int_{\bar{D}} \alpha(\mathbf{x}) \nabla_{\mathbf{y}} u(\mathbf{x}, \mathbf{y}) \nabla_{\mathbf{y}} v(\mathbf{x}, \mathbf{y}) \mathrm{d} \mathbf{y} \mathrm{d} \sigma_{\mathbf{x}} \\
& +\int_{\partial \bar{D}} \int_{\partial \bar{D}} \alpha(\mathbf{x}) \alpha(\mathbf{y}) u(\mathbf{x}, \mathbf{y}) v(\mathbf{x}, \mathbf{y}) \mathrm{d} \sigma_{\mathbf{y}} \mathrm{d} \sigma_{\mathbf{x}}
\end{aligned}
$$

and the linear form $L: H_{m i x}^{1}(\bar{D} \times \bar{D}) \rightarrow \mathbb{R}$ is

$$
\begin{aligned}
L(v):=\int_{\partial \bar{D}} \int_{\partial \bar{D}} \operatorname{Cor}_{\kappa}(\mathbf{x}, \mathbf{y})\left\{h(\mathbf{x})-\nabla_{\Gamma} \bar{u}(\mathbf{x}) \nabla_{\Gamma, \mathbf{x}}\right\} \\
\cdot\left\{h(\mathbf{y})-\nabla_{\Gamma} \bar{u}(\mathbf{y}) \nabla_{\Gamma, \mathbf{y}}\right\} v(\mathbf{x}, \mathbf{y}) \mathrm{d} \sigma_{\mathbf{y}} \mathrm{d} \sigma_{\mathbf{x}} .
\end{aligned}
$$

Theorem 2. The variational problems (8), (9), and (10) are uniquely solvable provided that $\alpha(\mathbf{x}) \not \equiv 0$.

Proof. The standard theory of Robin boundary value problems yields the existence of constants $0<c_{E} \leq c_{S}<\infty$ such that it holds

$$
c_{E}\|u\|_{H^{1}(\bar{D})}^{2} \leq a(u, u), \quad a(u, v) \leq c_{S}\|u\|_{H^{1}(\bar{D})}\|v\|_{H^{1}(\bar{D})}
$$

for all $u, v \in H^{1}(\bar{D})$. Thus, we conclude

$$
c_{E}^{2}\|u\|_{H_{\text {mix }}^{1}(\bar{D} \times \bar{D})}^{2} \leq A(u, u), \quad A(u, v) \leq c_{S}^{2}\|u\|_{H_{m i x}^{1}(\bar{D} \times \bar{D})}\|v\|_{H_{m i x}^{1}(\bar{D} \times \bar{D})}
$$

for all $u, v \in H_{m i x}^{1}(\bar{D} \times \bar{D})$ by a tensor product argument since the bilinear form $A(\cdot, \cdot)$ is derived from $a(\cdot, \cdot)$ via tensorization. The Lax-Milgram theorem implies finally the assertion.

Remark 2. If $\alpha(\mathbf{x}) \equiv 0$, then we arrive at the Neumann boundary value problem and obtain thus the ellipticity of $a(\cdot, \cdot)$ only in the space $\bar{H}^{1}(\bar{D}):=H^{1}(\bar{D}) \backslash \mathbb{R}$ and that of $A(\cdot, \cdot)$ in the space $\bar{H}_{m i x}^{1}(\bar{D} \times \bar{D}):=\bar{H}^{1}(\bar{D}) \otimes \bar{H}^{1}(\bar{D})$. Consequently, unique solvability of the variational problems (8), (9), and (10) is obtained in these energy spaces. 


\section{Solving tensor product boundary value problems}

\subsection{An abstract view on the linearization approach}

The linearization of a linear second order elliptic boundary value problem with respect to a given input parameter $\kappa(\omega)$ involves the associated derivative $\delta u(\omega) \in$ $\mathscr{H}(D)$. It is generally given by a boundary value problem

$$
\mathscr{A} \delta u(\omega)=f(\omega) \text { on } D,
$$

where $\mathscr{A}: \mathscr{H}(D) \rightarrow \mathscr{H}^{\prime}(D)$ denotes a linear, second order elliptic partial differential operator which is defined on a domain $D \subset \mathbb{R}^{n}$. Typically one might think of $\mathscr{H}(D)$ being a Sobolev space with dual $\mathscr{H}^{\prime}(D)$. Moreover, the random input parameter linearly enters the right hand side $f(\omega) \in \mathscr{H}^{\prime}(D)$ since the mapping $\kappa(\omega) \mapsto \delta u(\omega)$ is linear.

The two-point correlation $\operatorname{Cor}_{\delta u} \in \mathscr{H}_{\text {mix }}(D \times D):=\mathscr{H}(D) \otimes \mathscr{H}(D)$, which pops up in the asymptotic expansions (6), is given by the tensor product problem

$$
(\mathscr{A} \otimes \mathscr{A}) \operatorname{Cor}_{\delta u}=\operatorname{Cor}_{f} \quad \text { on } D \times D .
$$

Especially it holds $\operatorname{Cor}_{f} \in \mathscr{H}_{\text {mix }}^{\prime}(D \times D)=\mathscr{H}^{\prime}(D) \otimes \mathscr{H}^{\prime}(D)$.

In the following, we give an overview on the efficient solution of partial differential equations with the tensor product operator $\mathscr{A} \otimes \mathscr{A}$ on the product of the physical domain $D \times D$ such as (11). Various concepts are available to overcome the curse of dimension which is already observed in this moderate dimensional situation.

\subsection{Sparse tensor product spaces}

The starting point of the definition of sparse tensor product spaces for the Sobolev space $\mathscr{H}_{\text {mix }}(D \times D)$ are traditional and widely used multilevel hierarchies

$$
V_{0} \subset V_{1} \subset V_{2} \subset \cdots \subset \mathscr{H}(D)
$$

where $\operatorname{dim}\left(V_{j}\right) \sim 2^{j n}$. Then, appropriate complement spaces

$$
W_{0}:=V_{0}, \quad W_{j}:=V_{j} \ominus V_{j-1}, \quad j>0
$$

are chosen to derive the multiscale decomposition

$$
V_{J}=W_{0} \oplus W_{1} \oplus \cdots \oplus W_{J}
$$

In general, such complement spaces are defined by hierarchical bases like e.g. wavelet or multilevel bases, see [5] and the references therein. The sparse tensor product space $\widehat{V}_{J} \subset \mathscr{H}_{\text {mix }}(D \times D)$ is finally given via the complementary spaces according to 


$$
\widehat{V}_{J}=\bigoplus_{j+j^{\prime} \leq J} W_{j} \otimes W_{j^{\prime}}=\bigoplus_{j=0}^{J} V_{j} \otimes W_{J-j} .
$$

The sparse tensor product space $\widehat{V}_{J}$ possesses only $\mathscr{O}\left(2^{J n} J\right)$ degrees of freedom which is much less than the $\mathscr{O}\left(2^{2 J n}\right)$ degrees of freedom of the full tensor product space $V_{J} \otimes V_{J}$. However, the approximation power of the sparse tensor product space and the full tensor product space are essentially (i.e., except for logarithmic factors) identical if extra smoothness in terms of Sobolev spaces with dominating mixed derivative is given [5].

\subsection{Sparse multilevel frames}

In the meantime, the construction of wavelets on fairly general domains and surfaces is well understood [26, 29, 42]. However, the construction is expensive and the wavelets have large supports, particularly on complicated geometries. Therefore, other sparse tensor product approximations have been developed. In [17, 28], the sparse tensor product approximation has been performed via multilevel frames. The frame construction is based on the BPX-preconditioner (see e.g. $[4,10,35]$ ) and related generating systems (see e.g. $[16,17,19,20]$ ).

By rewriting the sparse tensor product space (13) according to

$$
\widehat{V}_{J}=\sum_{j+j^{\prime} \leq J} V_{j} \otimes V_{j^{\prime}}
$$

it is obvious that the collection of tensor products of the basis functions in $V_{j}$ can be used to represent the functions in $\widehat{V}_{J}$. It has been shown in [28] that this collection forms a frame for the sparse tensor product space provided that the basis functions are appropriate normalized.

The discretization of boundary value problems by frames and the solution of operator equations in frame coordinates is well understood and quite similar to the basis case, cf. e.g. [7, 9, 41]. The algorithms developed in [38], especially the applications of tensor product operators, can be extended to multilevel frames. It turns out that, in order to efficiently solve boundary value problems of the type (11), it suffices to provide standard multigrid hierarchies and associated finite elements together with prolongations and restrictions, see [22, 28].

\subsection{Combination technique}

Consider the tensor product boundary value problem (11). With respect to the ansatz spaces (12), we define the associated complement spaces by 


$$
W_{j}:=\left(P_{j}-P_{j-1}\right) \mathscr{H}(D) \subset V_{j}
$$

with $P_{j}: \mathscr{H}(D) \rightarrow V_{j}$ being the Galerkin projection associated with the operator $\mathscr{A}$. Then, the Galerkin system decouples due to Galerkin orthogonality. Namely, it holds

$$
\left((\mathscr{A} \otimes \mathscr{A}) v_{i, i^{\prime}}, w_{j, j^{\prime}}\right)_{L^{2}(D \times D)}=0 \text { for all } v_{i, i^{\prime}} \in W_{i} \otimes W_{i^{\prime}}, w_{j, j^{\prime}} \in W_{j} \otimes W_{j^{\prime}}
$$

provided that $i \neq j$ or $i^{\prime} \neq j^{\prime}$. As a consequence, the Galerkin solution $\widehat{\operatorname{Cor}}_{\delta u, J}$ to (11) in the sparse tensor product space (13) can be written as

$$
\widehat{\operatorname{Cor}}_{\delta u, J}=\sum_{j=0}^{J}\left(p_{j, J-j}-p_{j, J-j-1}\right) \in \bigoplus_{j=0}^{J} V_{j} \otimes W_{J-j}=\widehat{V}_{J}
$$

where $p_{j, j^{\prime}}$ denotes the Galerkin solution of (11) in the full (but small) tensor product space $V_{j} \otimes V_{j^{\prime}}$, cf. [25]. If the differential operator has not the form (11), then the combination technique induces an approximation error. Related error estimates have been derived in $[21,30,36]$.

\subsection{Low-rank approximation}

A rank- $r$ approximation of a given function $\operatorname{Cor}_{f} \in L^{2}(D \times D)$ is defined by

$$
\operatorname{Cor}_{f}(\mathbf{x}, \mathbf{y}) \approx \operatorname{Cor}_{f, r}(\mathbf{x}, \mathbf{y}):=\sum_{\ell=1}^{r} a_{\ell}(\mathbf{x}) b_{\ell}(\mathbf{y})
$$

with certain functions $a_{\ell}, b_{\ell} \in L^{2}(D)$. Inserting such a low-rank approximation in the tensor product boundary value problem (11) leads to the representation

$$
\operatorname{Cor}_{\delta u}=\left(\mathscr{A}^{-1} \otimes \mathscr{A}^{-1}\right) \operatorname{Cor}_{f} \approx\left(\mathscr{A}^{-1} \otimes \mathscr{A}^{-1}\right) \operatorname{Cor}_{f, r}=\sum_{\ell=1}^{r}\left(\mathscr{A}^{-1} a_{\ell}\right) \otimes\left(\mathscr{A}^{-1} b_{\ell}\right),
$$

i.e., the tensor product boundary value problem is reduced to $2 r$ simple boundary value problems on the domain $D$.

This approach has firstly been proposed in [15] for $m$-fold tensor product problems and right hand sides of tensor product type. In the case of the second moment analysis in uncertainty quantification, we find the special situation that $\mathrm{Cor}_{f}$ is symmetric and positive semi-definite. Thus, the pivoted Cholesky decomposition can be used to efficiently compute the low-rank approximation to the right hand side, see $[23,24]$. 


\section{Finite element discretization}

\subsection{Parametric finite elements}

For the application of multilevel techniques, we shall define a nested sequence of finite dimensional trial spaces

$$
V_{0} \subset V_{1} \subset \cdots \subset V_{j} \subset \cdots \subset H^{1}(\bar{D}) .
$$

In general, due to our smoothness assumptions on the domain, we have to deal with non-polygonal domains. To realize the multiresolution analysis (14) we will use parametric finite elements.

Let $\triangle$ denote the reference simplex in $\mathbb{R}^{n}$. We assume that the domain $\bar{D}$ is partitioned into a finite number of patches

$$
\operatorname{clos}(\bar{D})=\bigcup_{k} \tau_{0, k}, \quad \tau_{0, k}=\gamma_{k}(\triangle), \quad k=1,2, \ldots, M,
$$

where each $\gamma_{k}: \triangle \rightarrow \tau_{0, k}$ defines a diffeomorphism of $\triangle$ onto $\tau_{0, k}$. The intersection $\tau_{0, k} \cap \tau_{0, k^{\prime}}, k \neq k^{\prime}$, of the patches $\tau_{0, k}$ and $\tau_{0, k^{\prime}}$ is either $\emptyset$, or a lower dimensional face. The parametric representation is supposed to be globally continuous which means that the diffeomorphisms $\gamma_{i}$ and $\gamma_{i^{\prime}}$ coincide at common patch interfaces except for orientation. A mesh of level $j$ on $\bar{D}$ is then induced by regular subdivisions of depth $j$ of $\triangle$ into $2^{j n}$ simplices. This generates the $2^{j n} M$ curved elements $\left\{\tau_{j, k}\right\}$. An illustration of such a triangulation is found in Fig. 1.

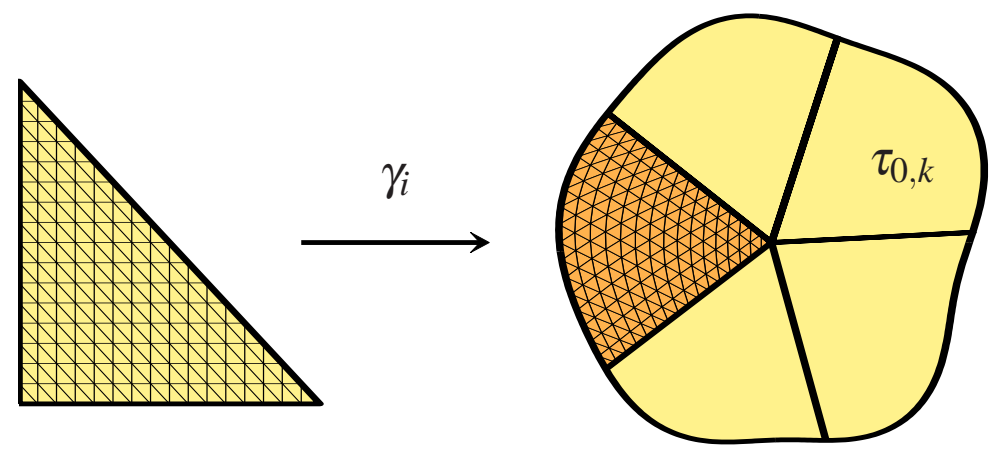

Fig. 1 Construction of parametric finite elements

The ansatz functions $\Phi_{j}=\left\{\varphi_{j, k}: k \in \Delta_{j}\right\}$ are finally defined via parameterization, lifting continuous piecewise linear Lagrangian finite elements from $\triangle$ to the domain $\bar{D}$ by using the mappings $\gamma_{i}$ and gluing across patch boundaries. Setting $V_{j}=\operatorname{span} \Phi_{j}$ yields (14), where $\operatorname{dim} V_{j} \sim 2^{j n}$. 


\subsection{Galerkin discretization}

We shall be concerned with Galerkin's method for solving the variational problems (8), (9), and (10). To this end, we define first the system matrix

$$
\mathbf{A}_{j}:=\left(\nabla \Phi_{j}, \nabla \Phi_{j}\right)_{L^{2}(\bar{D})}+\left(\alpha \Phi_{j}, \Phi_{j}\right)_{L^{2}(\partial \bar{D})}
$$

Then, the Galerkin solution

$$
\bar{u}_{j}=\sum_{k \in \Delta_{j}} u_{j, k} \varphi_{j, k}=\Phi_{j} \mathbf{u}_{j} \in V_{j}
$$

of the variational formulation (8) is derived from the linear system of equations

$$
\mathbf{A}_{j} \mathbf{u}_{j}=\mathbf{f}_{j}, \quad \text { where } \quad \mathbf{f}_{j}:=\left(f, \Phi_{j}\right)_{L^{2}(\bar{D})}+\left(g, \Phi_{j}\right)_{L^{2}(\partial \bar{D})} .
$$

The solution of this equation (16) by multigrid accelerated finite element methods is straightforward and along the lines of the standard literature, see e.g. [2, 3]. Therefore, we will skip all the details here.

The shape derivative $\delta u=\delta u[\kappa]$, given by (9), is approximated in a similar way: we seek

$$
\delta u_{j}=\sum_{k \in \Delta_{j}} v_{j, k} \varphi_{j, k}=\Phi_{j} \mathbf{v}_{j} \in V_{j}
$$

such that

$$
\mathbf{A}_{j} \mathbf{v}_{j}=\mathbf{g}_{j}, \quad \text { where } \quad \mathbf{g}_{j}:=\left(\kappa h, \Phi_{j}\right)_{L^{2}(\partial \bar{D})}+\left(\kappa \nabla_{\Gamma} \bar{u}, \nabla_{\Gamma} \Phi_{j}\right)_{L^{2}(\partial \bar{D})} .
$$

Likewise to the mean field equation, the solution of (17) is straightforward.

For the combination technique, we need to compute certain Galerkin approximations

$$
p_{j, j^{\prime}}=\sum_{k \in \Delta_{j}} \sum_{k^{\prime} \in \Delta_{j^{\prime}}} w_{(j, k),\left(j^{\prime}, k^{\prime}\right)}\left(\varphi_{j, k} \otimes \varphi_{j^{\prime}, k^{\prime}}\right)=\left(\Phi_{j} \otimes \Phi_{j^{\prime}}\right) \mathbf{w}_{j, j^{\prime}}
$$

to the two-point correlation $\operatorname{Cor}_{\delta u}(10)$ in the full tensor product space $V_{j} \otimes V_{j^{\prime}}$. They are obtained from the following linear system of equations

$$
\left(\mathbf{A}_{j} \otimes \mathbf{A}_{j^{\prime}}\right) \mathbf{w}_{j, j^{\prime}}=\mathbf{h}_{j, j^{\prime}}
$$

Here, the right hand side is given by

$$
\begin{aligned}
\mathbf{h}_{j, j^{\prime}} & :=\left(\operatorname{Cor}_{\kappa}(h \otimes h), \Phi_{j} \otimes \Phi_{j^{\prime}}\right)_{L^{2}(\partial \bar{D} \times \partial \bar{D})} \\
& -\left(\operatorname{Cor}_{\kappa}\left(\nabla_{\Gamma} \bar{u} \otimes h\right), \nabla_{\Gamma} \Phi_{j} \otimes \Phi_{j^{\prime}}\right)_{L^{2}(\partial \bar{D} \times \partial \bar{D})} \\
& -\left(\operatorname{Cor}_{\kappa}\left(h \otimes \nabla_{\Gamma} \bar{u}\right), \Phi_{j} \otimes \nabla_{\Gamma} \Phi_{j^{\prime}}\right)_{L^{2}(\partial \bar{D} \times \partial \bar{D})} \\
& +\left(\operatorname{Cor}_{\kappa}\left(\nabla_{\Gamma} \bar{u} \otimes \nabla_{\Gamma} \bar{u}\right), \nabla_{\Gamma} \Phi_{j} \otimes \nabla_{\Gamma} \Phi_{j^{\prime}}\right)_{L^{2}(\partial \bar{D} \times \partial \bar{D})} .
\end{aligned}
$$


The iterative solution of the tensor product problem (18) is of optimal complexity if the tensor product of the BPX-preconditioner [4] is applied.

\subsection{Combination technique}

According to Subsection 4.4, the combination technique amounts to solving all the Galerkin systems (18) which are needed to determine the expression

$$
\widehat{\operatorname{Cor}_{\delta u, J}}=\sum_{j=0}^{J}\left(p_{j, J-j}-p_{j, J-j-1}\right) \in \widehat{V}_{J}
$$

For the implementation of the combination technique, we have thus to explain how to efficiently compute the right hand side (19) to the linear system of equations (18). To this end, we shall introduce some notation first.

Let the index set $\Delta_{j}^{\partial \bar{D}} \subset \Delta_{j}$ denote the indices which belong to finite element functions at the boundary $\partial \bar{D}$ and set $\varphi_{j, k}^{\partial \bar{D}}:=\left.\varphi_{j, k}\right|_{\partial \bar{D}}$ for all $k \in \Delta_{j}^{\partial \bar{D}}$. Then, setting $\nabla_{0}^{\partial \bar{D}}:=\Delta_{0}^{\partial \bar{D}}$ and $\nabla_{j}:=\Delta_{j}^{\partial \bar{D}} \backslash \Delta_{j-1}^{\partial \bar{D}}$ for $j>0$, the hierarchical basis in the trace space $\left.V_{J}\right|_{\partial \bar{D}}$ is given by $\bigcup_{j=0}^{J}\left\{\varphi_{j, k}^{\partial \bar{D}}\right\}_{k \in \nabla_{j}^{\partial \bar{D}} \text {. We replace the two-point correlation function }}$ Cor $_{\kappa}$ by its piecewise linear sparse grid interpolant

$$
\widehat{\operatorname{Cor}}_{\kappa, J}=\left.\sum_{j+j^{\prime} \leq J} \sum_{k \in \nabla_{j}^{\partial \bar{D}}} \sum_{k^{\prime} \in \nabla_{j^{\prime}}^{\partial \bar{D}}} \gamma_{(j, k),\left(j^{\prime}, k^{\prime}\right)}\left(\varphi_{j, k}^{\partial \bar{D}} \otimes \varphi_{j^{\prime}, k^{\prime}}^{\partial \bar{D}}\right) \subset \widehat{V}_{J}\right|_{\partial \bar{D} \times \partial \bar{D}}
$$

which can be computed in optimal complexity (see [5]). Thus, the right hand side $\mathbf{h}_{j, j^{\prime}}$ becomes

$$
\mathbf{h}_{j, j^{\prime}}=\sum_{\ell+\ell^{\prime} \leq J}\left(\mathbf{M}_{j, \ell} \otimes \mathbf{M}_{j^{\prime}, \ell^{\prime}}\right)\left[\gamma_{(\ell, k),\left(\ell^{\prime}, k^{\prime}\right)}\right]_{k \in \nabla_{j}^{\partial \bar{D}}, k^{\prime} \in \nabla_{j^{\prime}}^{\partial \bar{D}}}
$$

where the matrices $\mathbf{M}_{j, j^{\prime}}, 0 \leq j, j^{\prime} \leq J$, are given by

$$
\mathbf{M}_{j, j^{\prime}}=\left[\left(\varphi_{j^{\prime}, k^{\prime}}^{\partial \bar{D}} h, \varphi_{j, k}\right)_{L^{2}(\partial \bar{D})}+\left(\varphi_{j^{\prime}, k^{\prime}}^{\partial \bar{D}} \nabla_{\Gamma} \bar{u}, \nabla_{\Gamma} \varphi_{j, k}\right)_{L^{2}(\partial \bar{D})}\right]_{k \in \Delta_{j}, k^{\prime} \in \nabla_{j^{\prime}}^{\partial \bar{D}}} \cdot
$$

The expression (20) can be evaluated in essentially optimal complexity by applying the matrix-vector multiplication from [28]. In particular, by using prolongations and restrictions, the matrices $\mathbf{M}_{j, j^{\prime}}$ are needed only in the situation $j=j^{\prime}$. Thus, the overall computational complexity of the combination technique is essentially linear in the number $\left|\Delta_{J}\right|$ of finite element functions on $\bar{D}$. 


\subsection{Low-rank approximation}

The piecewise linear interpolant of the two-point correlation Cor $_{\kappa}$ in the trace space $\left.\left(V_{j} \otimes V_{j}\right)\right|_{\partial \bar{D} \times \partial \bar{D}}$ is given by

$$
\operatorname{Cor}_{\kappa, j}=\sum_{k, k^{\prime} \in \Delta_{j}^{\partial \bar{D}}} \operatorname{Cor}_{\kappa}\left(\mathbf{x}_{j, k}, \mathbf{x}_{j^{\prime}, k^{\prime}}\right)\left(\varphi_{j, k}^{\partial \bar{D}} \otimes \varphi_{j, k^{\prime}}^{\partial \bar{D}}\right) .
$$

Here, $\mathbf{x}_{j, k} \in \partial \bar{D}$ denotes the node which belongs to the finite element basis function $\left.\varphi_{j, k}^{\partial \bar{D}} \in V_{j}\right|_{\partial \bar{D}}$. We shall thus compute a low-rank approximation of the matrix

$$
\mathbf{C}=\left[\operatorname{Cor}_{\kappa}\left(\mathbf{x}_{j, k}, \mathbf{x}_{j^{\prime}, k^{\prime}}\right)\right]_{k, k^{\prime} \in \Delta_{j}^{\partial \bar{D}}} \approx \mathbf{C}_{r}=\sum_{i=1}^{r} \kappa_{i} \kappa_{i}^{T}
$$

by the pivoted Cholesky decomposition. Afterwards, we just have to compute all the local shape derivatives $\delta u$ in the directions $\sum_{k \in \Delta_{j}^{\partial D}} \kappa_{i, k} \varphi_{j, k}^{\partial \bar{D}}$ via (17). Thus, having the low-rank approximation (21) at hand, the complexity to compute $\operatorname{Cor}_{\delta u, j}$ is $\mathscr{O}\left(r\left|\Delta_{j}\right|\right)$. Note here that, in accordance with [18, 39], the rank $r$ hinges on the smoothness of the underlying two-point correlation $\operatorname{Cor}_{\kappa}$.

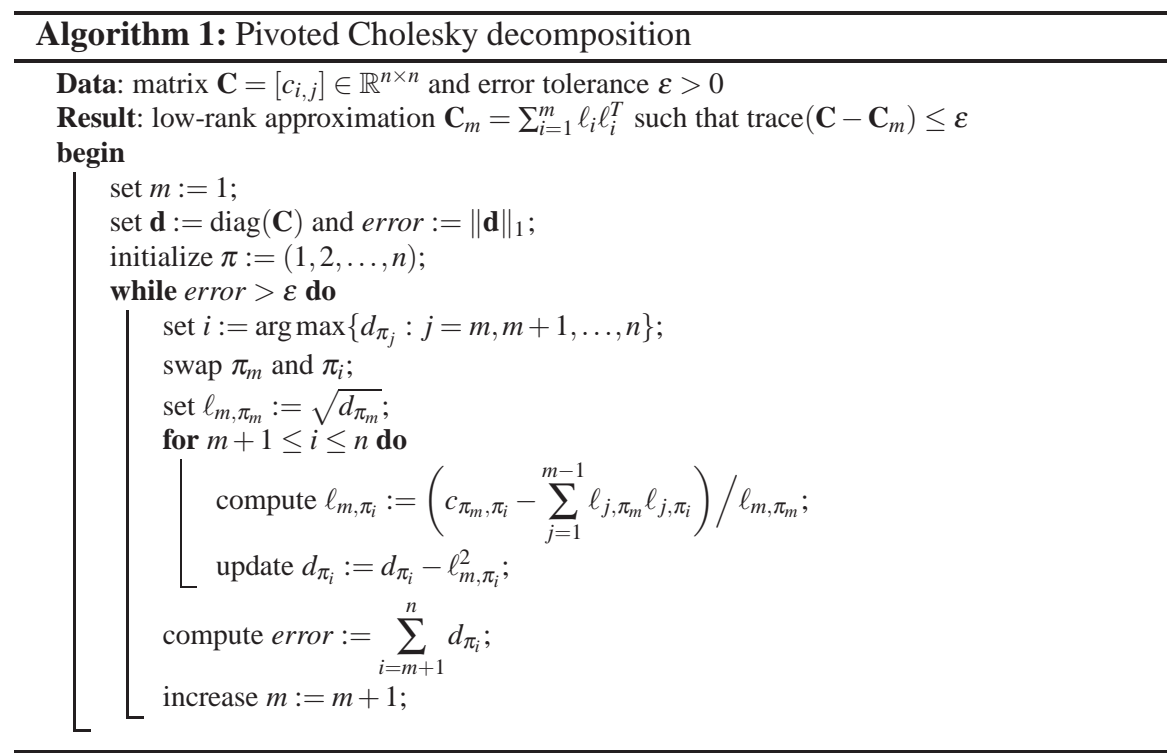

The pivoted Cholesky decomposition is a purely algebraic approach which is quite simple to implement, see Algorithm 1. It produces a low-rank approximation of $\mathbf{C}$ for any given precision $\varepsilon>0$ where the approximation error is rigorously con- 
trolled in the trace norm. A rank- $r$ approximation is computed in $\mathscr{O}\left(r^{2} n\right)$ operations, where $n$ denotes the matrix dimensions, that is $n=\left|\Delta_{j}^{\partial \bar{D}}\right|$. Exponential convergence rates in $r$ are proven under the assumption that the eigenvalues of $\mathbf{C}$ exhibit a sufficiently fast exponential decay, see [24]. Numerical experiments given there show that the pivoted Cholesky decomposition in general converges optimally in the sense that the rank $r$ is bounded by the number of terms required for the spectral decomposition of $\mathbf{C}$ to get the error $\varepsilon$.

\section{Numerical results}

\subsection{Model verification}

We present some numerical tests to demonstrate our theoretical predictions. Let $\bar{D}=\left\{\mathbf{x} \in \mathbb{R}^{2}:\|\mathbf{x}\|<1\right\}$ be the unit disk. We parametrize the boundary $\partial \bar{D}$ by polar coordinates

$$
\bar{\gamma}:[0,2 \pi] \rightarrow \partial \bar{D}, \quad s \mapsto \bar{\gamma}(s):=\left[\begin{array}{l}
\cos (s) \\
\sin (s)
\end{array}\right] .
$$

Correspondingly, the boundary $\partial D_{\varepsilon}(\omega)$ of the random domain $D_{\varepsilon}(\omega)$ can be expressed via the perturbed parametrization

$$
\gamma(s, \omega):=\bar{\gamma}(s)+\varepsilon \kappa(s, \omega)\left[\begin{array}{c}
\cos (s) \\
\sin (s)
\end{array}\right] .
$$

Herein, we assume that the random perturbation is given by

$$
\kappa(s, \omega):=\sum_{k=0}^{5} a_{k}(\omega) \cos (k s)+b_{k}(\omega) \sin (k s)
$$

with random coefficients $a_{k}(\omega)$ and $b_{k}(\omega)$ which are equally distributed in $[-1,1]$ and mutually stochastically independent. This results in the two-point correlation function

$$
\operatorname{Cor}_{\kappa}(s, t)=\frac{1}{3} \sum_{k=0}^{5} \cos (k s) \cos (k t)+\sin (k s) \sin (k t) .
$$

For our numerical experiments, we vary $0 \leq \varepsilon \leq 0.05$. Even though $\varepsilon$ is small, the perturbation is considerably large since the norm $\|\kappa(\omega)\|_{C^{2,1}([0,2 \pi])}$ might become large.

On the above defined random domain $D_{\varepsilon}(\omega)$, we consider the Robin boundary value problem (1) with $f(\mathbf{x}) \equiv 1, \alpha(\mathbf{x}) \equiv 1$, and $g(\mathbf{x}) \equiv 0$. For a given value of $\varepsilon$, we determine first the expectation and the variance of the random solution by a Monte Carlo method, using $M=25000$ samples. Note that the triangulation hast to be constructed for each sample in order to resolve the random domain. To evaluate the sample mean and variance, we interpolate each solution to a fixed quadrangular 
grid on the disk $K=\left\{\mathbf{x} \in \mathbb{R}^{2}:\|\mathbf{x}\| \leq 0.7\right\}$ with radius 0.7 which lies always in the interior of the random domain $D_{\varepsilon}(\omega)$. The result of the Monte Carlo simulation is then compared with the solution of our deterministic model. Here, we used the pivoted Cholesky decomposition since the two-point correlation (22) is of finite rank $r=11$.
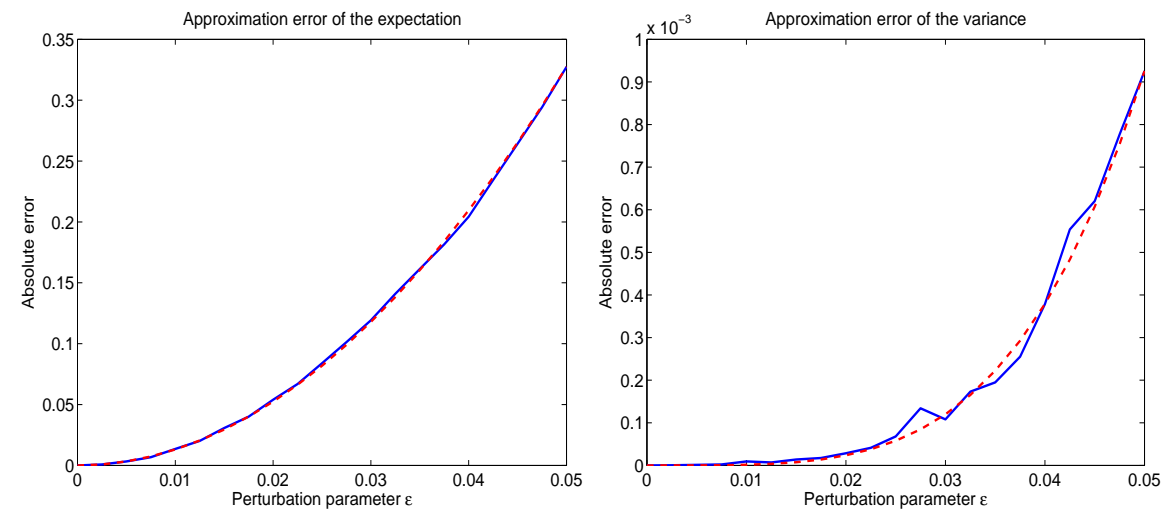

Fig. 2 Asymptotic behaviour with respect to the perturbation parameter $\varepsilon$ in the case of the expectation (left plot) and in the case of the variance (right plot).

In Figure 2, one finds the absolute difference between the mean (left plot) and variance (right plot) of the Monte Carlo simulation and the deterministic approach. To be on save ground, we repeated the comparison five times and computed the average of the differences. We observe that the difference behaves like $\mathscr{O}\left(\varepsilon^{2}\right)$ for the expectation (left plot) and like $\mathscr{O}\left(\varepsilon^{4}\right)$ for the variance (right plot) as indicated by the dashed lines. Hence, in this example, the asymptotic behaviour of the expectation with respect to the perturbation parameter $\varepsilon$ is as predicted by Theorem 1 . But the the asymptotic behaviour of the variance with respect to the perturbation parameter $\varepsilon$ is even one order better than predicted.

In Figure 3, we visualized the approximate moments computed by the Monte Carlo simulation (first row of Figure 3) and by the deterministic approach (second row of Figure 3) in the specific case $\varepsilon=0.025$. The difference between both approaches are found in the last row of Figure 3 . The relative difference in the mean has the order of magnitude $10^{-3}$ while the relative difference in the variance has the order of magnitude $10^{-2}$.

\subsection{A correlation kernel of arbitrary smoothness}

We shall next compare the low-rank approximation with the combination technique based sparse grid approach. To this end, we choose the same input data as before 


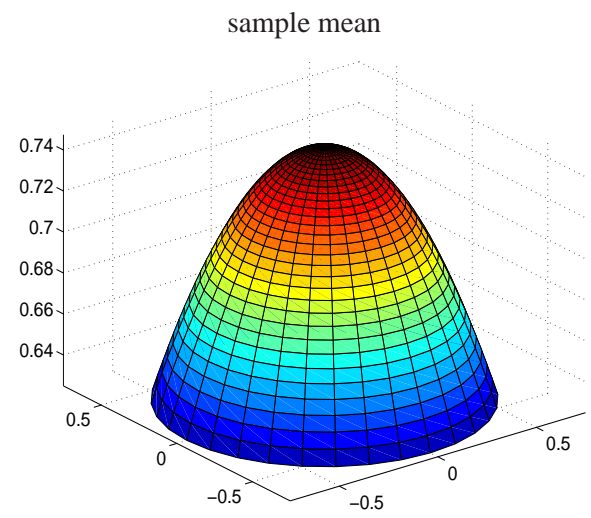

approximate mean

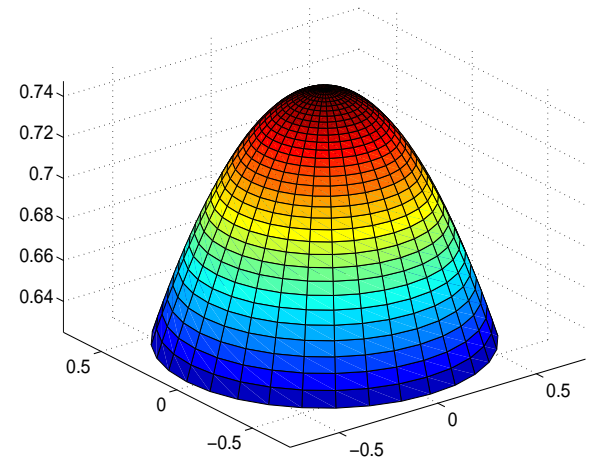

error of the expectation

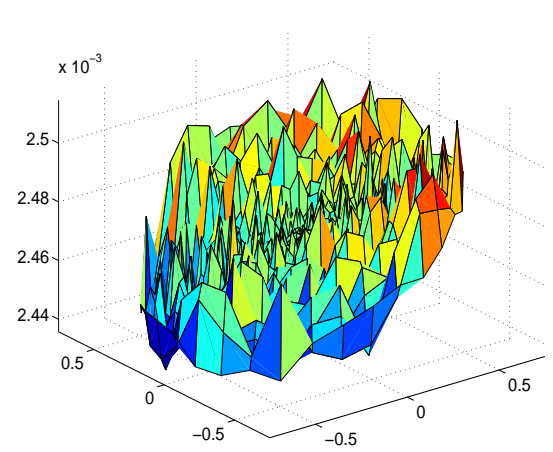

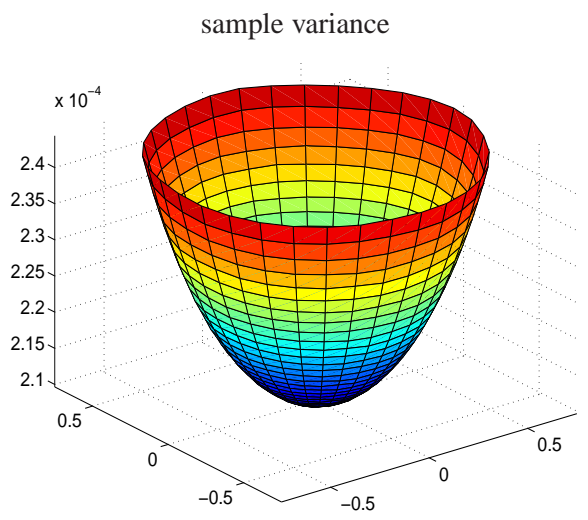

approximate variance

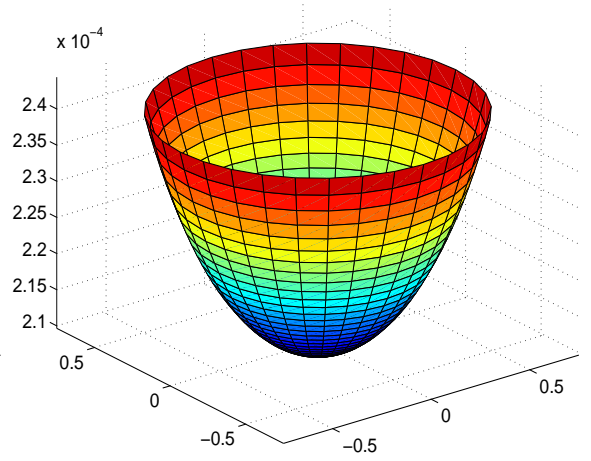

error of the variance

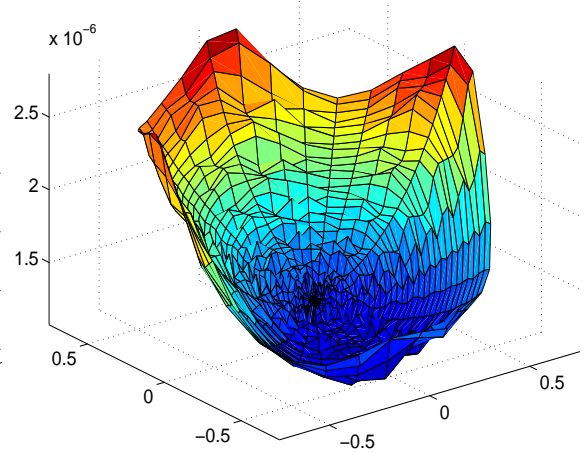

Fig. 3 Sample mean and variance (first row) versus the deterministic mean and variance (second row) in the case of $\varepsilon=0.025$. The differences are found in the last row. 
but the Gaussian kernel

$$
k(r)=\exp \left(-\frac{r^{2}}{\ell^{2}}\right), \quad r=\|\gamma(s)-\gamma(t)\|
$$

instead of the kernel (22). The Gaussian kernel is of arbitrary smoothness for any given correlation length $\ell>0$. In particular, the eigenvalues of the associated Hilbert-Schmidt operator decay double-exponentially (see e.g. [39]). In our numerical tests, we vary the correlation length according to $\ell=1,1 / 2,1 / 4,1 / 8$.

We compute a reference solution on a very fine level and compare the solutions of both approaches with respect to lower levels with this reference solution. The results are plotted in Figure 4, where the left plot shows the relative error of the variance versus the discretization level and the right plot shows the related computing times versus the discretization level. Note that on level 10, there are about 2 million finite elements.
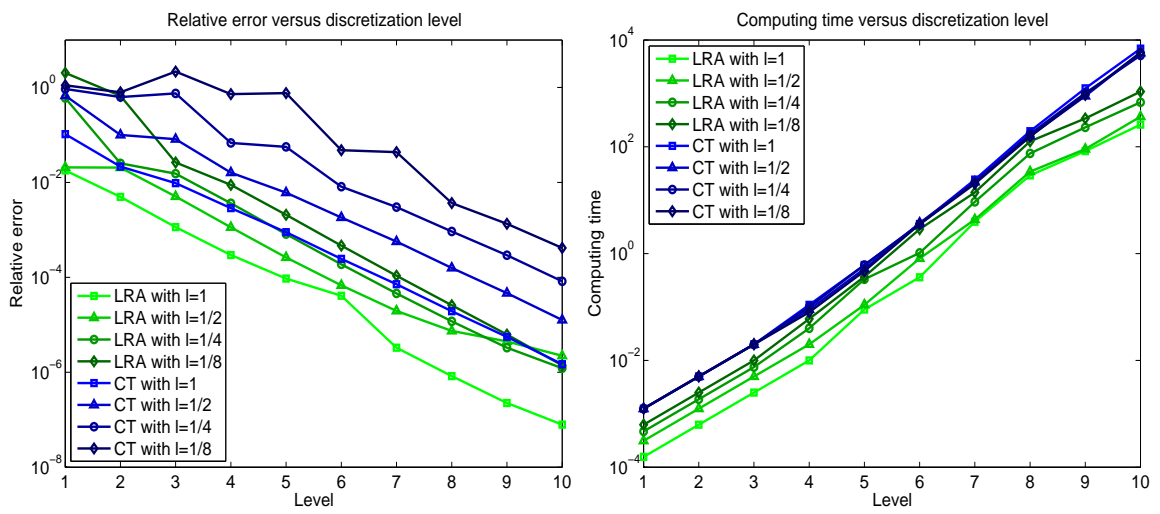

Fig. 4 Accuracy (left plot) and computing times (right plot) in the case of the Gaussian kernel.

It is observed that both, the convergence rates (left plot of Figure 4) and the computing times (right plot of Figure 4), scale identically for both approaches. The relative errors of both approaches increase when the correlation length decreases. The approximation errors of the low-rank approximation (green lines) are, however, a certain factor lower than the related approximation errors of the sparse grid method (blue lines). Also the computing times of the low-rank approximation (green lines) are a certain factor lower than the related computing times of the sparse grid approach (blue lines). Nevertheless, the computing times with respect to the sparse grid approach are essentially independent of the correlation length $\ell$ while the computing times of the low-rank approximation increase in $\ell$ as the rank increases. 


\subsection{A correlation kernel of finite smoothness}

We finally compare the low-rank approximation with the combination technique in case of the Matérn kernel

$$
k_{3 / 2}(r)=\left(1+\frac{\sqrt{3} r}{\ell}\right) \exp \left(-\frac{\sqrt{3} r}{\ell}\right), \quad r=\|\gamma(s)-\gamma(t)\|
$$

which is of finite smoothness. The correlation length $\ell$ is again chosen to be $\ell=$ $1,1 / 2,1 / 4,1 / 8$. The computational set-up of our comparison is in complete analogy to that of Subsection 6.2.
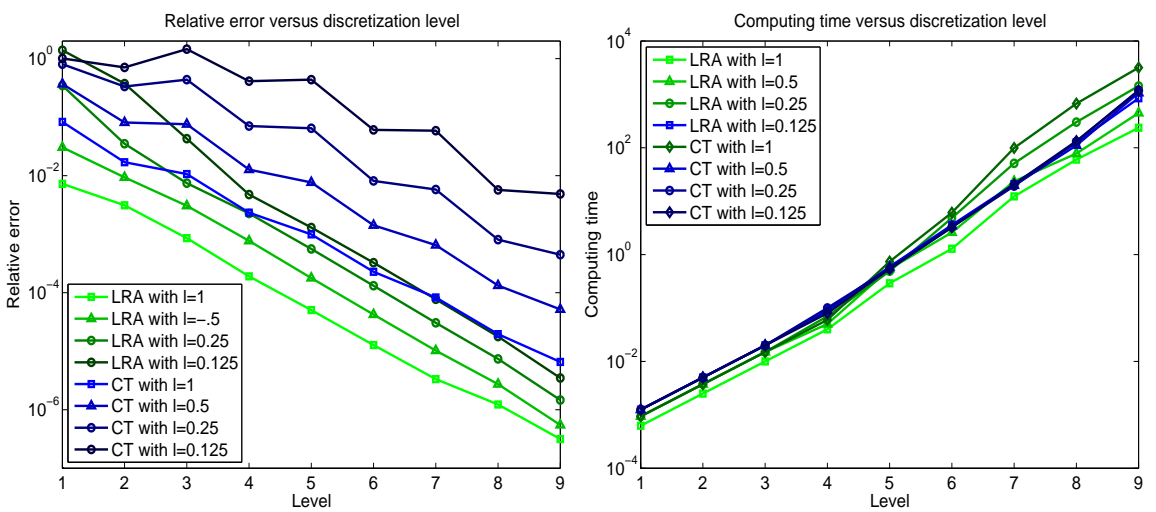

Fig. 5 Accuracy (left plot) and computing times (right plot) in the case of the Matern kernel.

In the left plot of Figure 5, we plotted the relative error of the variance versus the discretization level. Again, both approaches seem to produce the same convergence rates but the relative errors of the the low-rank approximation (green lines) are again a certain factor lower than relative error of the sparse grid approach (blue lines). Moreover, for a fixed discretization level, the relative error increases as the correlation length decreases.

In the right plot of Figure 5, the associated computing times are found. The computing times of the low-rank approximation (green lines) clearly depend on the correlation length. Whereas, in the case of the sparse grid approach, the computing times are independent of the correlation length. Additionally, one figures out of the plot that the computing times of the low-rank approximation seem to grow with a higher rate compared with the sparse grid approach. This corresponds to the theoretical predictions from [18]. Nevertheless, if one compares accuracy versus computing time, the low-rank approximation is still superior to the sparse grid approach. 


\section{Concluding remarks}

In this paper, we modeled and solved Robin boundary value problems on random domains. We derived deterministic equations for the expectation and variance of the associated random solution. The variance can be computed by means of a lowrank approximation or by the combination technique. By numerical experiments, we compare these two approaches. It turns out that for our specific examples the low-rank approximation performs better than the combination technique. However, the combination technique has the advantage that the memory requirements are independent on the given two-point correlation function. We emphasize that, in the present case of boundary value problems on random domains, the low-rank approximation needs only to be computed for an $(n-1)$-dimensional function (cf. (21)) whereas the combination technique is an $n$-dimensional approach. Nevertheless, we expect that, in the case of random coefficients (see [25]) or random loadings (see [38]), the combination technique performs much better in comparison with the low-rank approximation since there the low-rank approximation of a $n$-dimensional function is required.

\section{References}

1. Babuška, I., Nobile, F., Tempone, R.: Worst case scenario analysis for elliptic problems with uncertainty. Numer. Math. 101, 185-219 (2005)

2. Braess, D.: Finite elements. Theory, fast solvers, and applications in solid mechanics. Second edition. Cambridge University Press, Cambridge (2001)

3. Brenner, S.C., Scott, L.R.: The mathematical theory of finite element methods. Texts in Applied Mathematics, 15. Springer, New York (1994)

4. Bramble, J., Pasciak, J., Xu, J.: Parallel multilevel preconditioners. Math. Comput. 55, 1-22 (1990)

5. Bungartz, H.J., Griebel, M.: Sparse grids. Acta Numerica 13, 147-269 (2004)

6. Canuto, C., Kozubek, T.: A fictitious domain approach to the numerical solution of PDEs in stochastic domains. Numer. Math. 107, 257-293 (2007)

7. Christiansen, O.: An introduction to frames and Riesz bases. Applied and Numerical Harmonic Analysis. Birkhäuser Boston, Inc., Boston (2002)

8. Chernov, A., Schwab, C. First order $k$-th moment finite element analysis of nonlinear operator equations with stochastic data. to appear in Math. Comput. (2012)

9. Dahlke, S., Fornasier, M., Raasch, T., Stevenson, R., Werner, M. Adaptive frame methods for elliptic operator equations. The steepest descent approach. IMA J. Numer. Math. 27, 717-740 (2007)

10. Dahmen, W.: Wavelet and multiscale methods for operator equations. Acta Numerica 6, 55-228 (1997)

11. Deb, M.K., Babuška, I., Oden, J.T.: Solution of stochastic partial differential equations using Galerkin finite element techniques. Comput. Methods Appl. Mech. Eng. 190, 6359-6372 (2001)

12. Delfour, M., Zolesio, J.-P.: Shapes and geometries. SIAM, Philadelphia (2001)

13. Frauenfelder, P., Schwab, C., Todor, R.A.: Finite elements for elliptic problems with stochastic coefficients. Comput. Meth. Appl. Mech. Engrg. 194, 205-228 (2004)

14. Ghanem, R.G., Spanos, P.D.: Stochastic finite elements. A spectral approach. Springer, New York (1991) 
15. Grasedyck, L.: Existence and computation of a low Kronecker-rank approximant to the solution of a tensor system with tensor right-hand side. Computing 72, 247-265 (2004)

16. Griebel, M.: Multilevel algorithms considered as iterative methods on semidefinite systems. SIAM J. Sci. Comput. 15, 547-565 (1994)

17. Griebel, M.: Multilevelmethoden als Iterationsverfahren über Erzeugendensystemen. Teubner Skripten zur Numerik. B.G. Teubner, Stuttgart (1994)

18. Griebel, M., Harbrecht, H.: Approximation of two-variate functions: singular value decomposition versus regular sparse grids. Preprint 488, Berichtsreihe des SFB 611, Universität Bonn, Germany, 2010.

19. Griebel, M., Oswald, P.: On additive Schwarz preconditioners for sparse grid discretizations. Numer. Math. 66, 449-463 (1994)

20. Griebel, M., Oswald, P.: Tensor product type subspace splittings and multilevel iterative methods for anisotropic problems. Adv. Comput. Math. 4, 171-206 (1995)

21. Griebel, M., Schneider, M., Zenger, C.: A combination technique for the solution of sparse grid problems. In P. de Groen and R. Beauwens, editors, Iterative Methods in Linear Algebra. pp. 263-281, IMACS, Elsevier, North Holland (1992)

22. Harbrecht, H.: A finite element method for elliptic problems with stochastic input data. Appl. Numer. Math. 60, 227-244 (2010)

23. Harbrecht, H., Li, J.: A fast deterministic method for stochastic elliptic interface problems based on low-rank approximation. Research Report No. 2011-24, Seminar für Angewandte Mathematik, ETH Zürich (2011)

24. Harbrecht, H., Peters, M., Schneider, R.: On the low-rank approximation by the pivoted Cholesky decomposition. Appl. Numer. Math. 62, 428-440 (2012)

25. Harbrecht, H., Peters, M., Siebenmorgen, M.: Combination technique based $k$-th moment analysis of elliptic problems with random diffusion. Preprint 2011-02, Mathematisches Institut, Universität Basel, Switzerland (2011)

26. Harbrecht, H., Schneider, R.: Biorthogonal wavelet bases for the boundary element method. Math. Nachr. 269-270, 167-188 (2004)

27. Harbrecht, H., Schneider, R., Schwab, C.: Sparse second moment analysis for elliptic problems in stochastic domains. Numer. Math. 109, 167-188 (2008)

28. Harbrecht, H., Schneider, R., Schwab, C.: Multilevel frames for sparse tensor product spaces. Numer. Math. 110, 199-220 (2008)

29. Harbrecht, H., Stevenson, R.: Wavelets with patchwise cancellation properties. Math. Comput. 75, 1871-1889 (2006)

30. Hegland, M., Garcke, J., Challis, V.: The combination technique and some generalisations. Linear Algebra Appl. 420, 249-275 (2007)

31. Hiptmair, R., Li, J.: Shape derivatives in differential forms I. An intrinsic perspective. to appear in Ann. Mat. Pura Appl. (2012)

32. Matthies, H.G., Keese, A.: Galerkin methods for linear and nonlinear elliptic stochastic partial differential equations. Comput. Methods Appl. Mech. Engrg. 194, 1295-1331 (2005)

33. Mohan, P.S., Nair, P.B., Keane, A.J.: Stochastic projection schemes for deterministic linear elliptic partial differential equations on random domains. Internat. J. Numer. Methods Engrg. 85, 874-895 (2011)

34. Nouy, A., Chevreuil, M., Safatly, E.: Fictitious domain method and separated representations for the solution of boundary value problems on uncertain parameterized domains. Comput. Methods Appl. Mech. Engrg. 200, 3066-3082 (2011)

35. Oswald, P.: Multilevel finite element approximation. Theory and applications. Teubner Skripten zur Numerik. B.G. Teubner, Stuttgart (1994)

36. Pflaum, C., Zhou, A.: Error analysis of the combination technique. Numer. Math. 84, 327-350 (1999)

37. Protter, P.: Stochastic integration and differential equations. A new approach. Springer-Verlag, Berlin (1990)

38. Schwab, C., Todor, R.: Sparse finite elements for elliptic problems with stochastic loading. Numer. Math. 95, 707-734 (2003) 
39. Schwab, C., Todor, R.: Karhunen-Loéve approximation of random fields by generalized fast multipole methods. J. Comput. Phys. 217, 100-122 (2006)

40. Sokolowski, J., Zolesio, J.-P.: Introduction to shape optimization. Springer-Verlag, Berlin (1992)

41. Stevenson, R.: Adaptive solution of operator equations using wavelet frames. SIAM J. Numer. Anal. 41, 1074-1100 (2003)

42. Stevenson, R.: Composite wavelet bases with extended stability and cancellation properties. SIAM J. Numer. Anal. 45, 133-162 (2007)

43. Tartakovsky, D.M., Xiu, D.: Numerical methods for differential equations in random domains. SIAM J. Sci. Comput. 28, 1167-1185 (2006) 